Herzschr Elektrophys 2022 · 33:108-112 https://doi.org/10.1007/s00399-022-00838-y Angenommen: 12. Januar 2022 Online publiziert: 25. Januar 2022

(C) The Author(s), under exclusive licence to Springer Medizin Verlag $\mathrm{GmbH}$, ein Teil von Springer Nature 2022

\title{
Nachruf auf Prof. Dr. med. Dr. h.c. Berndt Lüderitz
}

\author{
*26.03.1940, † 20.11.2021
}

\author{
Thorsten Lewalter ${ }^{1} \cdot$ Christian Wolpert $^{2} \cdot$ Dietrich Pfeiffer $^{3} \cdot$ Carsten W. Israel $^{4}$. \\ Thomas Klingenheben ${ }^{5}$ \\ 'Peter Osypka Herzzentrum, Internistisches Klinikum München Süd, München, Deutschland \\ ${ }^{2}$ Klinikum Ludwigsburg, Ludwigsburg, Deutschland \\ ${ }^{3}$ Universität Leipzig, Leipzig, Deutschland \\ ${ }^{4}$ Ev. Klinikum Bethel, Bielefeld, Deutschland \\ ${ }^{5}$ Praxis für Kardiologie, Bonn, Deutschland
}

\section{Thorsten Lewalter}

Insgesamt 15 Jahre, von 1992-2007, war ich an der Bonner Klinik unter der Leitung von Prof. Lüderitz tätig; initial als jüngster Kollege und Arzt im Praktikum, zuletzt als stellvertretender Klinikdirektor, so dass ich unseren Chef aus sehr unterschiedlicher Perspektive erleben durfte. Da an dieser Stelle der persönlichen Erinnerung und der Anekdote als Form des Gedenkens an Prof. Lüderitz Raum gegeben wird, möchte ich dies in Form einiger persönlicher Erlebnisse tun.

Erste Frühbesprechung als neu eingestellter Arzt im Praktikum: Der Dienstarzt berichtet aus der Nacht: „Prof. Lüderitz, es ist etwas Schlimmes passiert. Wir haben ein MRT bei einem Patienten mit Herzschrittmacher durchgeführt!", Prof. Lüde-

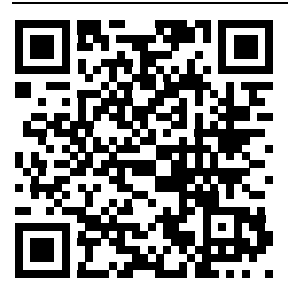

QR-Code scannen \& Beitrag online lesen

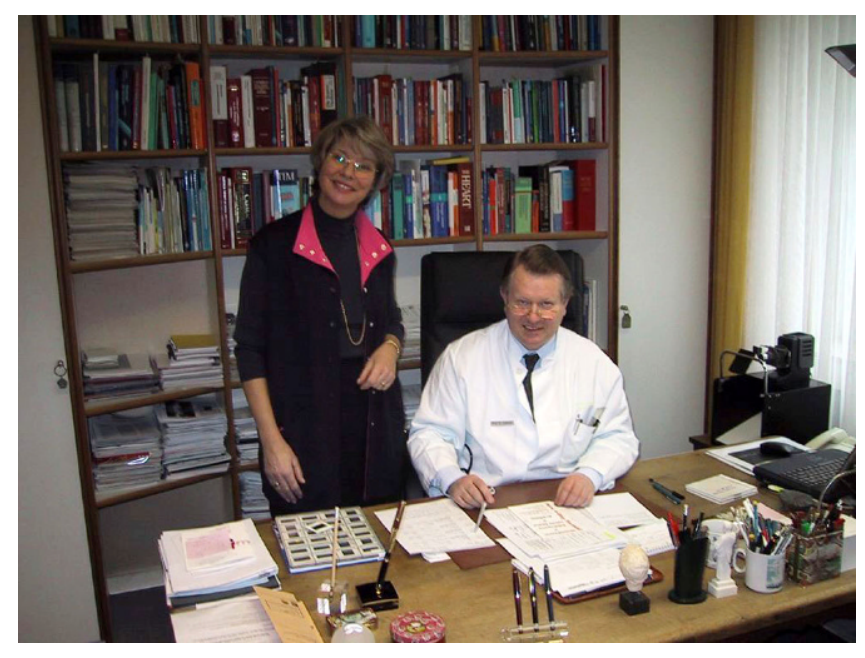

Abb. $1 \triangleleft$ Prof. Lüderitz in "typischer Position" am Schreibtisch des Direktors der Bonner Klinik; an seiner linken Seite seine langjährige Sekretärin Frau Schilling; an seiner rechten Seite eine Projektionsmöglichkeit für Diapositive, die Frau Schilling nach entsprechender Vorgabe anfertigte 

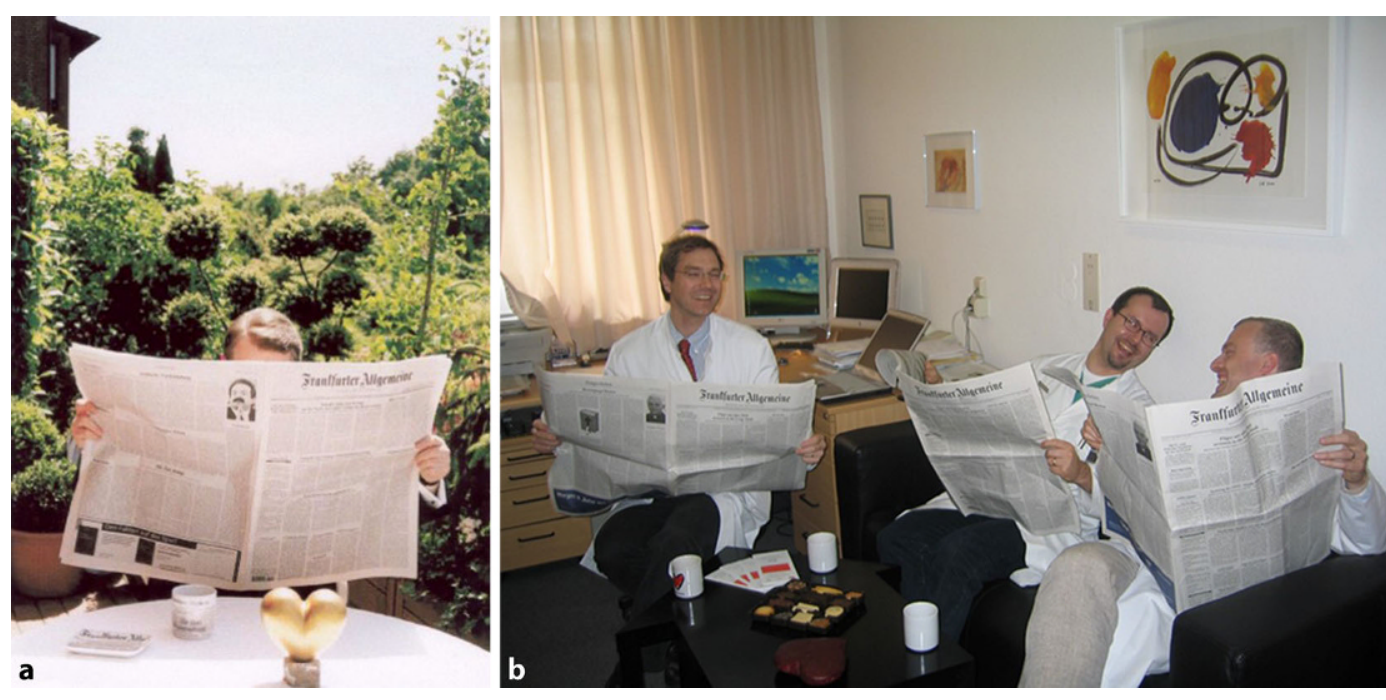

Abb. $2 \triangleleft$ a Die Frankfurter Allgemeine Zeitung spielte im Klinikalltag eine besondere Rolle. Dahinter steckt immer ein kluger Kopf ... b Zitat Prof. Lüderitz an seine Oberärzte (damals ausschließlich Männer): „Meine Herren, Ihr Patient liest Zeitung ... also müssen Sie es auch tun!". „Fühbesprechung" in der Medizinischen Universitätsklinik Bonn, die damaligen Oberärzte (von links T. Lewalter, K. Tiemann und J. Schwab)

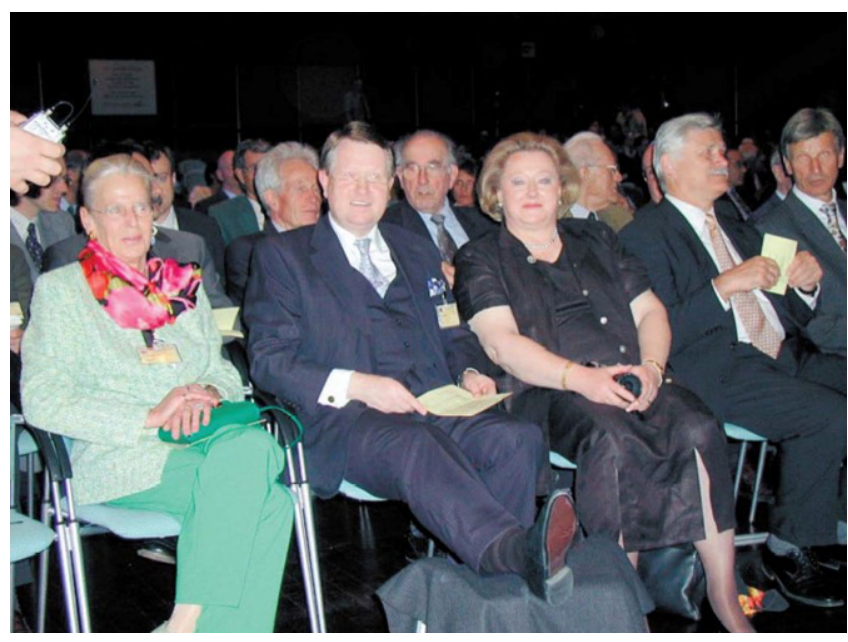

Abb. $3<$ Unverwüstlich, trotz Treppensturz und Knieoperation, immer in der ersten Reihe

ritz: „Und, was ist passiert?", Dienstarzt: „Nichts Besonderes, aber das MRT ist ja streng verboten bei Herzschrittmacherpatienten!“, Lüderitz: „Dann machen Sie noch 5 weitere Patienten und schreiben mal lhr erstes Abstract!"

Mein erster Einsatzort als Assistenzarzt: Station Martini - Privatstation! Einweisung durch Dr. Tebbenjohanns: Morgens um 7:30 Uhr kommt Prof. Lüderitz zur Visite; da muss man die Patienten aber alle schon kennen oder auch gesprochen haben, was in der Nacht vorgefallen war! Abends dann Visite ca. 20 Uhr mit dem Oberarzt. „Am Samstag und Sonntag reicht es, wenn du ca. 9 Uhr da bist, und es ist auch früher Schluss!". Nach ca. 8 Wochen Dienst, also unter der Woche von 7:15-ca. 20 Uhr und Präsenz am Samstag und Sonntag, nahm ich mir ein Herz und fragte Prof. Lüderitz, ob ich am kommenden Wochenende einmal frei haben könnte. Er sah mich kurz an und sagte: „Auf keinen Fall!“ und ging weiter. Am nächsten Tag kam der Leitende Oberarzt Prof. Manz zu mir und sagt: „Herr Lewalter, ich habe gehört, Sie haben Probleme ... ich weiß schon. Schreiben Sie einfach auf einen Zettel was Sie brauchen; meine Frau kauft für Sie ein." Damit war weiterhin die durchgehende Präsenz des Privatassistenten gewährleistet (• Abb. 1, 2 und 3 ).

Im Folgenden einige „bonmots" von Prof. Lüderitz. Beim Symposium „Klinische Kardiologie im neuen Jahrtausend" in Wiesbaden leitete er seinen Vortrag mit den Worten ein: „Ich darf im Hinblick auf meine Vorredner um Entschuldigung bitten, dass ich das ein oder andere Dia in deutscher Ausfertigung zeigen werde", was vor einem ausschließlich deutschsprachigen Publikum natürlich als eine feine Nadelspitze zu verstehen ist.
Prof. Lüderitz war ein unbestechlicher Leser und Korrektor von Texten. Dabei hatten korrekte Rechtschreibung und ein gewählter Ausdruck sowie Stil eine besondere Bedeutung. Zitat Prof. Lüderitz: „Herr Lewalter, haben Sie die vielen Rechtschreibfehler deswegen in Ihr Manuskript hineinfabriziert, um zu überprüfen, ob ich es überhaupt lese?"

Bonmot von Prof. Lüderitz zum Begriff der Schule in der Medizin: „Als Schule in der Medizin bezeichnet man eine Situation, in der einer - in aller Regel der Direktor der Klinik - etwas mit einer der Sachlage meist nicht angemessenen Festigkeit und Überzeugung vorgibt, die mehrere Andere - meistens Abhängige - oft gegen besseres Wissen aus fehlender Courage heraus nicht anzuzweifeln wagen."

\section{Christian Wolpert}

Es war 2016, als meine Kollegin Frau Peters, früher Oberarztsekretärin bei Professor Lüderitz in Bonn, seit 12 Jahren mit mir in Ludwigsburg tätig, sagte: „Herr Professor Lüderitz kommt mit nach Armenien! Er hat gesagt, ich soll für Sie gemeinsame Flüge von Stuttgart nach Yerevan buchen. Seine Frau bringt ihn dorthin. "Er macht tatsächlich ernst, dachte ich, und kommt mit nach Armenien zu unserem Elektrophysiologieprojekt (- Abb. 4). Wir flogen gemeinsam nach Yerevan, und auch 15 Jahre nach meinem Abschied aus Bonn war alles wie immer - nur anders. Entgegen meiner Befürchtung, und vielleicht auch, weil ich glaubte, seine Gedanken zumindest 


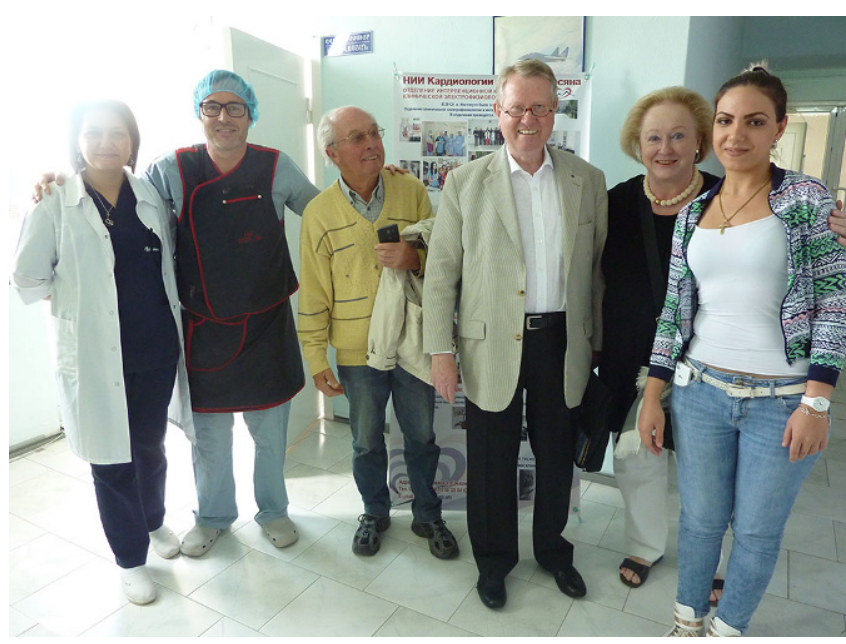

Abb. $4 \Delta$ Elektrophysiologieprojekt in Armenien. Christian Wolpert (2. von links) mit Berndt und Hedwig Lüderitz (3. und 2. von rechts) im Katheterlabor in Yerevan, Armenien

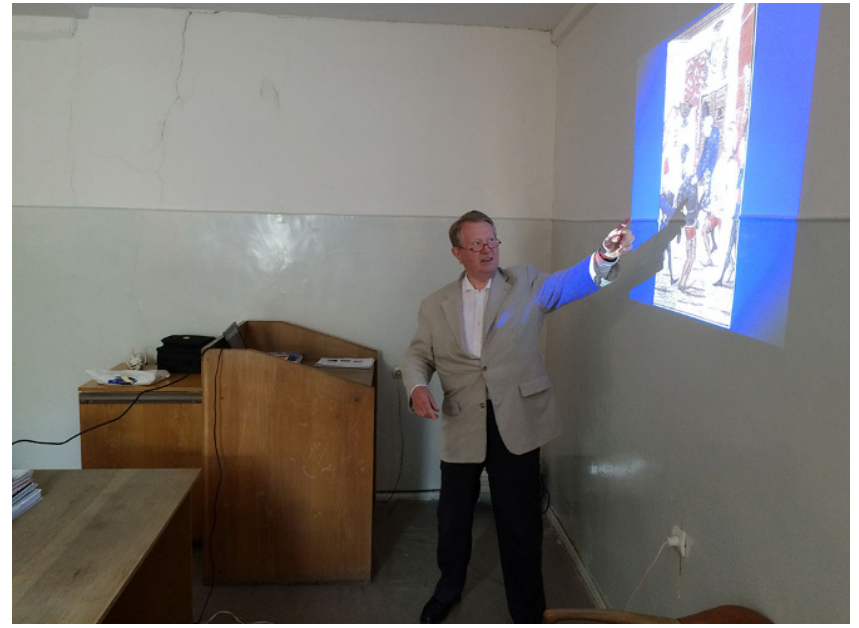

Abb. 5 \ Vortrag zur Geschichte der Herzrhythmusstörungen in Yerewan, Armenien in Bezug auf Abläufe, Termintreue, Dresscode, Sprache etc. lesen zu können, wurde es ein sehr lebhafter, verlängerter Ausflug ins Ungewisse. Sämtliche kleinen Verstöße meinerseits gegen die Etikette wurden mit kleinen versteckten Spitzen sehr zur Erheiterung meines Kollegen Norman Rüb quittiert. Es war erstaunlich, wie gut mein ehemaliger Chef mich kannte, wo doch früher der Abstand zwischen dem Direktor und dem Arzt im Praktikum um einiges größer war als heute. Mein Oberarzt Rüb und er verstanden sich auf Anhieb prächtig. Professor Lüderitz brachte inn mit vielen Anekdoten aus der Bonner Zeit regelmäßig zum Lachen, und sie hatten eine sehr unterhaltsame Zeit zusammen. Er ließ es sich nicht nehmen, bei den Ablationen und Operationen dabei zu sein und hatte große Freude daran, wenn die Bahn weg oder der "slow pathway" gefallen war. Klar war für mich auch, dass, wenn ich damals unter seiner Leitung nicht von Herrn Manz, Jung, Pfeiffer und anderen hätte lernen dürfen, wir mit diesem Projekt nicht hier wären. Professor Lüderitz hat uns immer "machen lassen". Insofern schloss sich hier der Kreis. Er hielt dann vor den armenischen Kollegen und der Fakultät seinen unvergesslichen Vortrag über die Geschichte der Herzrhythmusstörungen (- Abb.5), der mit viel Beifall bedacht wurde. Den Part des digitalen Diaschiebers - „Next slide please!" - übernahm in diesem Fall unser armenischer Fellow Dr. Ohanian. Ab mittags gaben wir Professor
Lüderitz frei, damit er seinem Interesse für die jüngere und auch ältere Geschichte nachgehen konnte ( $\mathbf{A b b}$. 6). Die geht in Armenien über weit mehr als 2000 Jahre und daher ist er auch ein Jahr später mit seiner Frau Hedwig zurückgekehrt, um die vielen interessanten Orte und Museen $z u$ besuchen, die er bei der ersten Reise nicht hatte sehen können. Beide haben das Land ebenso in ihr Herz geschlossen, wie wir es getan haben. Religion, Kunst, alte Schriften und die unendliche Gastfreundschaft haben seine Frau Hedwig und inn stark beeindruckt. In Marbach, der Schillerstadt, haben wir dann ein Jahr später noch einmal bei einem Tafelspitz unsere Fotos und Erinnerungen ausgetauscht. Da saß ich also mit meinem Chef und seiner Frau und schaute mir statt der Dias über den Atrioverter oder Vorhofrefraktärzeiten Bilder von Kirchen, Menschen und seltenen religiösen Schriften an. In nachfolgenden Jahren haben wir uns dann gelegentlich Zeitungsartikel oder Bilder über Armenien zugeschickt. Das hätte ich mir 1992 nie träumen lassen, und so habe ich Herrn Professor Lüderitz und seine Frau auch auBerhalb der Klinik kennenglernt.

\section{Dietrich Pfeiffer}

Berndt Lüderitz hatte für viele Lebenssituationen seine stereotypen Kommentare, die alle seine Mitarbeiter hundertmal gehört haben: Für inn musste die Klinik "auf Öl laufen“. Das fiel mir schwer: Ich war viel mehr an medizinischen Problemen in Diagnostik und Therapie interessiert und weniger an reibungslosen Abläufen. Wenn eine schwierige Verhandlung mit der Verwaltung oder im Fakultätsrat nicht nach seinem Wunsch verlaufen war, dann kommentierte er das mit dem Hinweis: „Wenn Sie einer Situation nicht gewachsen sind, dann gehen Sie einfach nicht hin!". Was hätte ich tun sollen? Ich musste hingehen, denn er hatte mich ja hingeschickt, und die Situation kannte ich doch zuvor nicht. Das Ende einer Besprechung wurde oft kommentiert mit dem Satz: "Gibt es sonst noch irgendwelche Hinweise, Aspekte oder Gesichtspunkte, die wir heute und hier lösen können? Wenn nicht, dann vertagen wir uns auf morgen 8:30 Uhr". Darin lag eine Botschaft: Sein Fokus lag auf von uns kurzfristig lösbaren Problemen, nicht auf der unbeeinflussbaren Großwetterlage. Neue Mitarbeiter bekamen ein paar Wochen Schonzeit, um "den Stallgeruch der Klinik anzunehmen", wobei "Stallgeruch" heute etwas euphemistisch als "Corporate Identity" bezeichnet würde. Seine Mitarbeiter sollten die klinikinterne Sicht der Dinge vertreten. Und die musste man erst erlernen. Hatte man einen interessanten Krankheitsverlauf erlebt, dann war er hartnäckig und wollte eine "Verdichtung in einem Manuskript" sehen. Meist erhielt man gleich einen Termin für die Vorlage des ersten Entwurfs, den er niemals vergaß. „Eine Klinik ist nicht so gut, wie der Chef es ist. Sie ist so gut, wie die Oberärz- 


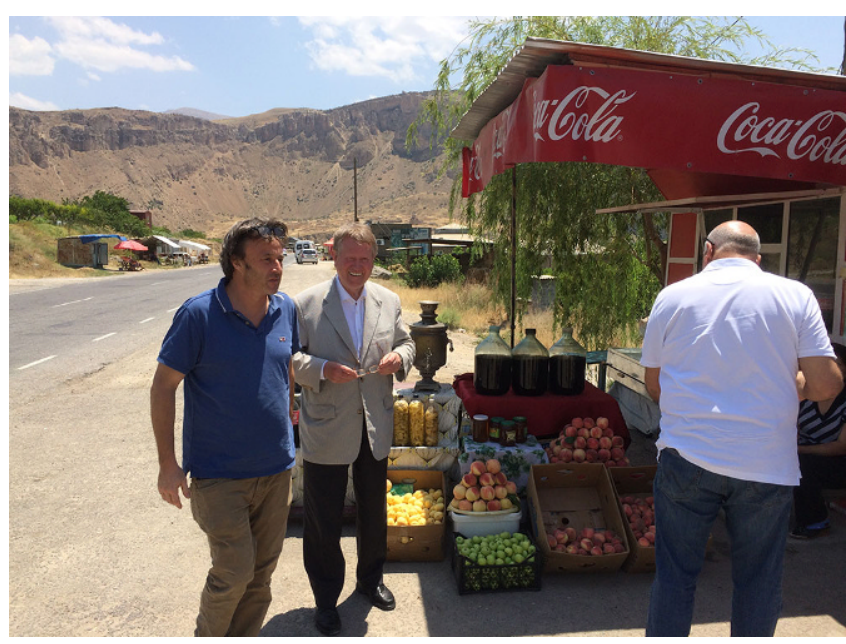

Abb. $6 \Delta$ Nach der Arbeit am Straßenkiosk in Armenien

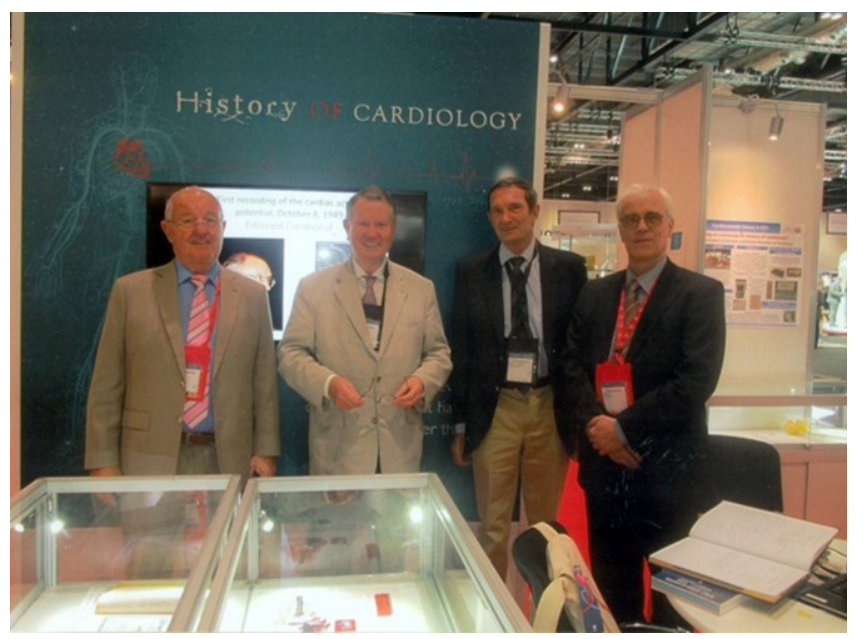

Abb. $8<$ Stand „History of Cardiology" anlässlich des Europäischen Kardiologenkongresses 2016 in Rom mit Otmar Pachinger (Innsbruck/A), Berndt Lüderitz (München), JeanJacques Monsuez (Vincennes/F) und Dietrich Pfeiffer (Leipzig)

te es sind." Dieser Satz war keine billige Wertschätzung der Mitarbeiter, es war der Hinweis, dass man nicht nur die eigene Reputation beschädigen kann, sondern immer gleichzeitig auch die Reputation der ganzen Klinik.

Ich habe erst viel später verstanden, dass in solchen Sätzen große Erfahrung in der Klinikleitung und sicher auch im Leben lag. Und ich kann nicht bestreiten, dass ich den einen oder anderen seiner stereotypen Sätze später selbst verwendet habe. Ärzte lernen im Klinikalltag dazu. Aber es bleibt ungewöhnlich, wenn Mitarbeiter noch Jahre nach ihrem Ausscheiden aus der Klinik Botschaften oft gehörter, aber nie wirklich verstandener Aussagen ihres früheren Chefs begreifen. Ich habe mich in den 25 Jahren nach meinem Ausscheiden aus der Bonner Universitätsklinik gelegentlich dabei ertappt, zu überlegen: Wie würde Berndt Lüderitz mit diesem Problem umgehen? Manchmal hat mir dieser Gedanke wirklich geholfen. Gelegentlich habe ich inn noch als Emeritus um einen Rat gebeten. Sein Tod hat mir diese Möglichkeit genommen, ich werde ihn vermissen (- Abb. 7 und 8).

\section{Carsten Israel}

Berndt Lüderitz war für mich in den 1980er Jahren eine der Lichtgestalten bei der Neubewertung der Antiarrhythmika vor dem Hintergrund der Ergebnisse der CAST-Studie, klassischer Arzt mit weißem Kittel (inklusive gestärktem Kragen) vor eindrucksvoller medizinischer Bücherwand und mit freundlichem Lächeln, das auch eine gewisse Strenge erahnen ließ. Ich konnte immer wieder seine unerreichte Fähigkeit genießen, schlagfertig und ausgesprochen pointiert, in geschliffenen Formulierungen auf Fortbildungsveranstaltungen vorzutragen oder später häufiger noch vorzusitzen. Der Humor, gerne gewürzt mit einer Prise Zynismus und Sarkasmus, hatte hohen Unterhaltungswert, insbesondere, wenn man gerade nicht selbst Ziel spöttischer Kommentare war. Ein besonders schönes jährliches Forum für Berndt Lüderitz' Humor waren die Pro- und Contra-Sitzungen auf den Rhythmologischen Expertentreffen in Berlin, wo er die Kontrahenten durchaus ermutigte, nicht unnötig auf verletzende Kommentare und einseitige Darstellungen zu verzichten. Nicht nur hier wird sein Fehlen sicher schmerzlich auffallen.

Persönlich verdanke ich Herrn Lüderitz (außer der Begutachtung meiner Habilitation), dass ich die Tätigkeit in Bielefeld in besonders angenehmer Perspektive betrachten konnte. Als ich gerade damit haderte, aus dem kosmopolitischen Frankfurt mit internationalem Flair und weltweiten Flugverbindungen in den schattigen Teutoburger Wald und eine Stadt ohne Mainufer gewechselt zu sein, kam Berndt Lüderitz auf mich zu: „Sie leben jetzt in Bielefeld? Sie Glücklicher! Ich bin in Herford aufs Gymnasium gegangen, und wenn wir am Samstag mal nach Bielefeld fahren durften - das war für uns wie eine Reise nach Paris, da gab es ja alles!"

Berndt Lüderitz wird mir mit seinem trockenen Humor, seinem verschmitzten 


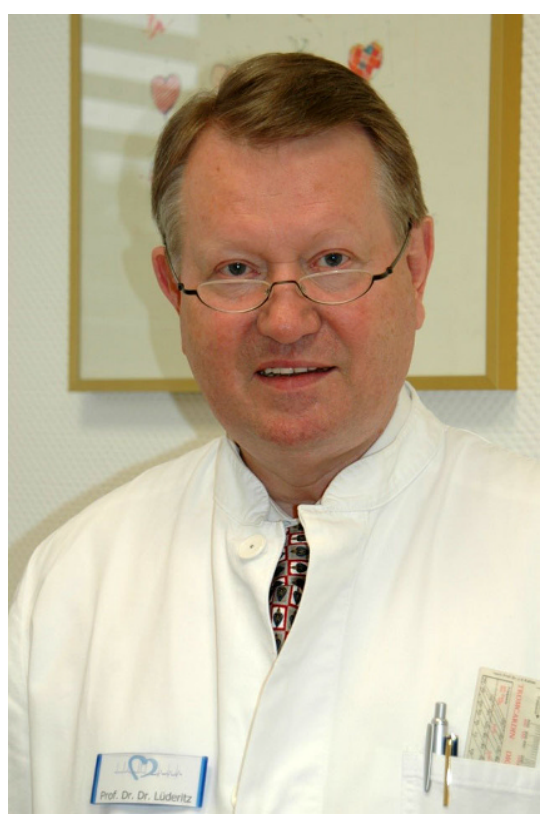

Abb. $9 \Delta$ Berndt Lüderitz in seinem Sprechzimmer in Bonn

Lächeln und seiner emeritären Altersmilde fehlen.

\section{Thomas Klingenheben}

Obwohl kein „Bonner Gewächs", kannte ich Professor Berndt Lüderitz natürlich bereits vor meiner Niederlassung in Bonn von etlichen wissenschaftlichen Veranstaltungen. Insbesondere sind mir einzelne Vorträge unter seinem Vorsitz in lebhafter Erinnerung geblieben, denn dies waren Gelegenheiten, bei denen man als Vortragender gut daran tat, für die Diskussion besonders gut mit geistigem Rüstzeug und auch einer gewissen Schlagfertigkeit gewappnet zu sein. Kurz nach seiner Emeritierung als Direktor der Klinik für Innere Medizin II (Kardiologie, Pneumologie) an der Rheinischen FriedrichWilhelms-Universität Bonn trat Professor Lüderitz 2005 in die Praxis für Kardiologie Bonn ein (-Abb.9), wo er noch für etliche Jahre seinen Patienten ein zugewandter Arzt gewesen ist und uns als hoch geschätzter und partnerschaftlicher Kollege durch seinen umfänglichen Erfahrungsschatz bereichert hat. Die anfängliche Sorge meinerseits, ob er sich in den Abläufen einer Praxis "an der kardiologischen Basis" überhaupt wohlfühlen würde, erwies sich ziemlich schnell als absolut unbegründet. Mit der ihm eigenen Fähigkeit, auf Stim-

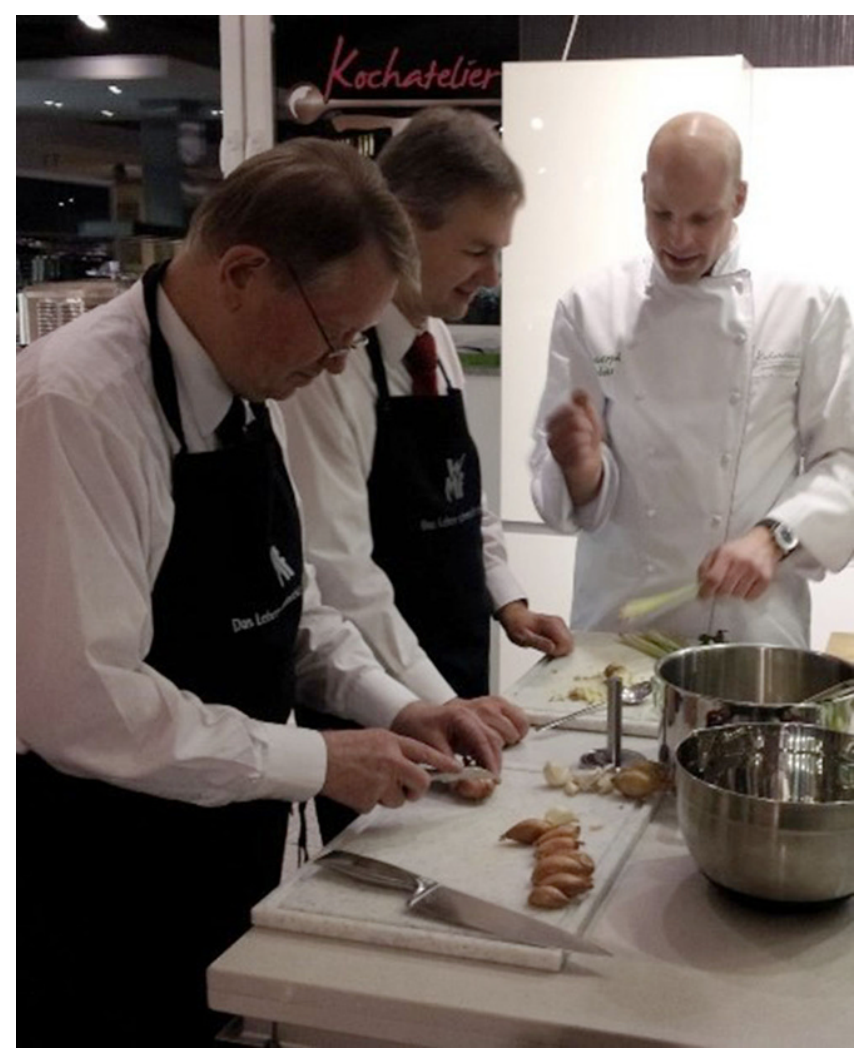

Abb. $10 \triangle$ „Maître de Cardiologie" trifft auf „Maître de Cuisine" im Advent 2008. Das exquisite 5-Gänge-Menü musste vor dem Verzehr erst durch eigener Hände Werk erarbeitet werden... mungen und neue Situationen einzugehen, hat Herr Lüderitz sich alsbald mit der Institution identifiziert. So wurde beispielsweise bereits in jedem Frühherbst obligat der geplante Termin der Weihnachtsfeier abgefragt, damit dieses Event nicht mit anderen wichtigen Dezember-Terminen (z. B. Rhythmologisches Expertengespräch Berlin) in Kollision geraten möge (- Abb. 10). Dass er die etwa ein Jahrzehnt dauernde gemeinsame Tätigkeit ziemlich genossen hat, zeigte auch folgender Ausspruch: „Herr Klingenheben, jetzt bin ich ja schon fast 2 Jahre hier in der Praxis, und wissen Sie, was mich wirklich überrascht: Wir haben uns ja noch nie gestritten! - Komisch, früher in der Klinik war das überhaupt kein Problem".

\section{Korrespondenzadresse}

\section{PD Dr. med. Carsten W. Israel}

Ev. Klinikum Bethel

Bielefeld, Deutschland

Carsten.Israel@evkb.de 